\title{
A SUFFICIENT CONDITION THAT A MONOTONE IMAGE OF THE THREE-SPHERE BE A TOPOLOGICAL THREE-SPHERE
}

\author{
O. G. HARROLD, JR. ${ }^{1}$
}

1. A continuous transformation of one space onto another is called monotone provided the complete inverse set for each point of the image space is connected. A monotone image of a circle is a simple closed curve or a point. A monotone image of a 2 -sphere is a configuration known as a cactoid, i.e. a peano space in which every true cyclic element is a topological 2-sphere. R. L. Moore has shown that if a monotone transformation of a 2 -sphere has the additional property that no inverse set separates the 2 -sphere, then the image space is again a topological 2-sphere or a point [3]. In the case of the threesphere, $S^{3}$, as one would expect, the situation is more complicated and extra conditions need to be imposed if the image space is to be expected to look like an $S^{3}$.

A recent example of R. H. Bing [1] shows that if a monotone transformation on $S^{3}$ has the property that for each point of the image the complement of the inverse image is an open 3-cell, the image may not be a topological $S^{3}$, thus answering a long standing conjecture. By studying this example and profiting by conversations with Professor Bing the author was led to the following theorem.

2. THEOREM 1. Let $M=f\left(S^{3}\right)$, where $f$ is a monotone, continuous map such that (i) if $Y=\left\{y \in M \mid f^{-1}(y)\right.$ does not reduce to a point $\}$, then given $y \in \bar{Y}$, and $\epsilon>0$, there is a topological 2-sphere $K$ in $S(y, \epsilon)$ separating $y$ and $M \backslash S(y, \epsilon)$ such that $K$ does not meet $\bar{Y}$. Then $M$ is a topological 3-sphere. ${ }^{2}$

Proof. Let $\epsilon_{1}>\epsilon_{2}>\cdots \rightarrow 0$ and $\sum \epsilon_{i}<+\infty$. The set $\bar{Y}$ is totally disconnected. Hence $\bar{Y}=Y_{1} \cup \ldots \cup Y_{n_{1}}$ where $Y_{i}$ is closed, ${ }^{3}$ $Y_{i} \cap Y_{j}=\square$ and $\delta\left(Y_{i}\right)<\epsilon_{1} / 4$. Suppose $\eta_{1}=\min \rho\left[Y_{i}, Y_{j}\right], i \neq j$. De-

Presented to the Society June 21, 1958; received by the Editors March 21, 1958.

1 Fellow of the John Simon Guggenheim Memorial Foundation. Research also supported by the National Science Foundation, NSF, G-2793.

$2 \bar{Y}=$ closure of $Y$.

${ }^{3} \delta(Y)$ represents the diameter of $Y, S(y, \epsilon)$ is the set of points each of whose distance from $y$ is less than $\epsilon$. The symbol $\rho$ represents the metric of the space concerned. It will be clear whether $\rho$ refers to $M$ or $S^{3}$ by noting in which space the sets are given.

If $K$ is a topological 2-sphere in $M \backslash \bar{Y}$, the complement of $\bar{Y}$ in $M$, Int $K$ $=f\left[\operatorname{Int} f^{-1}(K)\right]$. 
fine $\epsilon_{1}^{\prime}=\min \left(\epsilon_{1} / 4, \eta_{1} / 3\right)$. A finite number of topological 2-spheres $K_{1}^{\prime}, \cdots, K_{m_{1}}^{\prime}$ are found, by use of (i), such that for $i=1, \cdots, m_{1}$,

$$
\begin{aligned}
& \delta\left(K_{i}^{\prime}\right)<\epsilon_{1}^{\prime} ; \\
& U \text { Int } K_{i}^{\prime} \supset \bar{Y} ; \\
& K_{i}^{\prime} \cap \bar{Y}=\square .
\end{aligned}
$$

The first set of operations is designed to replace the spheres $K_{1}^{\prime}, \cdots, K_{m_{1}}^{\prime}$ by a set $\tilde{K}_{1}^{\prime}, \cdots, \tilde{K}_{p_{1}}^{\prime}$ that enjoy properties similar to (1), (2), (3) and the further requirement

$$
\tilde{K}_{i}^{\prime} \cap \tilde{K}_{j}^{\prime}=\square,
$$

$$
i \neq j \text {. }
$$

The set of spheres $\tilde{K}_{1}^{\prime}, \cdots, \tilde{K}_{p_{1}}^{\prime}$ may be found as follows. Since $f^{-1}$ is topological on $K_{i}^{\prime}, L_{i}=f^{-1}\left(K_{i}^{\prime}\right)$ is a topological 2-sphere. Since $\rho\left[L_{i}, f^{-1} \bar{Y}\right]>0$, we may apply the Bing approximation theorem [2] to find a polyhedral 2 -sphere $P_{i}$ as near $L_{i}$ as we please so that $P_{i}$ contains in its interior precisely those components of $f^{-1} \bar{Y}$ that are interior to $L_{i}$. By doing this for each $i$, we obtain a set of polyhedral 2-spheres

$$
P_{1}, \cdots, P_{n_{1}} \text {. }
$$

It may be supposed further that $P_{i} \cap P_{j}$ is a finite collection (possibly null) of pairwise disjoint simple closed curves, none of which may be removed by an arbitrarily small deformation of $P_{i}$ or $P_{j}$. In addition,

$$
\begin{aligned}
U \text { Int } P_{i} & \supset f^{-1}(\bar{Y}) . \\
P_{i} \cap f^{-1}(\bar{Y}) & =\square .
\end{aligned}
$$

We first describe how to find a set of polyhedral 2 -spheres $\widetilde{P}_{1}, \cdots, \widetilde{P}_{p_{1}}$ such that conditions $\left(2^{\prime}\right),\left(3^{\prime}\right)$ and the following hold

$$
\widetilde{P}_{i} \cap \widetilde{P}_{j}=\square .
$$

Suppose $C_{1}, \cdots, C_{q}$ are the components of $P_{1} \cap P_{2}$. If $q=1$, let $C_{1}$ divide $P_{1}$ into $U_{1}, V_{1}$ and $C_{1}$ divide $P_{2}$ into $U_{2}, V_{2}$. Then $P_{1}$ and the closure of the component ( $V_{2}$ say) of $P_{2} \backslash C_{1}$ in the exterior of $P_{1}$ together with the appropriate $\operatorname{disk}\left(U_{1}\right.$ or $\left.V_{1}\right)$ gives a pair of 2 -spheres $P_{1}, P_{2}^{\prime}$ that covers the same part of $f^{-1}(\bar{Y})$ that $P_{1} \cup P_{2}$ does, neither $P_{1}$ nor $P_{2}^{\prime}$ meets $f^{-1}(\bar{Y})$ and, by a slight deformation $P_{1} \cap P_{2}^{\prime}=\square$.

If $q>1$, at least one of $C_{1}, \cdots, C_{q}$, say $C_{1}$, will not separate $C_{2}, \cdots, C_{q}$ on $P_{1}$. (Of course $C_{1}$ may separate $C_{2}, \cdots, C_{q}$ on $P_{2}$, but that is irrelevant.) By replacing $P_{2}$ by 2 new polyhedral 2 -spheres meeting along a disk on $P_{1}$, we again have covered the same part of 
$f^{-1}(\bar{Y})$ and by a pair of slight deformations obtain 3 polyhedral 2spheres

$$
P_{1}, P_{2}^{\prime}, P_{2}^{\prime \prime}
$$

such that the number of components of $P_{1} \cap P_{2}^{\prime}$ or $P_{1} \cap P_{2}^{\prime \prime}$ is less than $q$.

Continuing, we obtain, after a finite number of such operations a collection of polyhedral 2-spheres

$$
\widetilde{P}_{1}, \cdots, \widetilde{P}_{p_{1}}
$$

such that

$$
\begin{aligned}
U \text { Int } \tilde{P}_{i} \supset f^{-1}(\bar{Y}) . \\
\tilde{P}_{i} \cap f^{-1}(\bar{Y})=\square . \\
\tilde{P}_{i} \cap \tilde{P}_{j}=\square, i \neq j .
\end{aligned}
$$

Define $\widetilde{K}_{i}=f\left(\widetilde{P}_{i}\right)$. We note that under the steps made in forming $\tilde{P}_{i}$, or, correspondingly, $\tilde{K}_{i}$, that the diameters of the spheres replacing $K_{j}$ may be greater than that of $K_{j}$. However, since $\epsilon_{1}^{\prime}<\eta_{1}(1 / 3)$, the definition of $\eta_{1}$ and the triangle inequality show that $\delta\left(\widetilde{K}_{i}\right)$ $<3 \epsilon_{1} / 4<\epsilon_{1}$. Hence $\tilde{K}_{1}, \cdots, \widetilde{K}_{p_{1}}$ satisfy

$$
\begin{aligned}
\delta\left(K_{i}\right) & <\epsilon_{1}: \\
U \text { Int } K_{1}^{\prime} & \supset \bar{Y}: \\
\tilde{K}_{i}^{\prime} \cap \bar{Y} & =\square, \\
\tilde{K}_{i}^{\prime} \cap \tilde{K}_{j}^{\prime} & =\square, i \neq j .
\end{aligned}
$$

To $\epsilon_{2}>0$, write $Y_{i}=Y_{i, 1} \cup \ldots \cup Y_{i, n_{2}}$, where $Y_{i, j}$ is closed, $Y_{i, j} \cap Y_{i, j^{\prime}}=\square$ and $\delta\left(Y_{i, j}\right)<\epsilon_{2} / 4$. Put

$$
\eta_{2}=\min \rho\left[Y_{i, j}, Y_{i^{\prime}, j^{\prime}}\right], \rho\left[Y_{i, j}, \bigcup_{1}^{p_{1}} \tilde{K}_{i}\right] \text {. }
$$

Let $\epsilon_{2}^{\prime}<\epsilon_{2} / 4, \eta_{2} / 3$. By the hypotheses (i) there is a finite collection of topological 2-spheres in $M, K_{1}^{2}, \cdots, K_{m_{2}}^{2}$ such that

$$
\begin{array}{r}
\delta\left(K_{i}^{2}\right)<\epsilon_{2}^{\prime}: \\
\bigcup_{1}^{m_{2}} \operatorname{Int} K_{i}^{2} \supset \bar{Y}: \\
K_{i}^{2} \cap \bar{Y}=\square .
\end{array}
$$

By the choice of $\epsilon_{2}^{\prime}, K_{\imath}^{2} \cap \tilde{K}_{j}^{1}=\square$. By modifications of the $K_{1}^{2}, \cdots, K_{m_{2}}^{2}$ precisely as above at the first stage we arrive at another set of spheres $\tilde{K}_{1}^{2}, \cdots, \tilde{K}_{p_{2}}^{2}$ such that 


$$
\begin{aligned}
\delta\left(\tilde{K}_{i}^{2}\right)<\epsilon_{2}, \\
\bigcup_{1}^{p_{2}} \text { Int } K_{i}^{2} \supset \bar{Y}, \\
K_{i}^{2} \cap \bar{Y}=\square, \\
K_{i}^{2} \cap K_{j}^{2}=\square, i \neq j \\
K_{i}^{1} \cap K_{j}^{2}=\square .
\end{aligned}
$$

The general step is now clear.

To $\epsilon_{n}>0$ we find a finite set of topological 2-spheres

$$
\tilde{K}_{1}^{n}, \cdots, \tilde{K}_{p_{n}}^{n}
$$

such that

$$
\begin{aligned}
& \delta\left(\tilde{K}_{i}^{n}\right)<\epsilon_{n}, \\
& \bigcup_{1}^{p_{n}} \text { Int } \widetilde{K}_{i}^{n} \supset \bar{Y}, \\
& \tilde{K}_{i}^{n} \cap \bar{Y}=\square, \\
& \widetilde{K}_{i}^{n} \cap \tilde{K}_{j}^{n}=\square, i \neq j \\
& \widetilde{K}_{i}^{n} \cap \widetilde{K}_{j}^{p}=\square, p<n, \text { all } i, j .
\end{aligned}
$$

3. Let $F_{1}^{\prime}, \cdots, F_{p_{1}}^{\prime}$ be $p_{1}$ disjoint cubes (topological 2-spheres) with centers on the $x$-axis and faces parallel to the co-ordinate planes. We take the cubes congruent to one another for convenience. Let $F_{1}^{2}, \cdots, F_{p_{2}}^{2}$ be a similar set of cubes of smaller size so that

if and only if

$$
F_{i}^{2} \subset \operatorname{Int} F_{j}^{\prime}
$$

$$
K_{i}^{2} \subset \operatorname{Int} K_{j}^{\prime} .
$$

Continuing, for each $n$ we have

$$
F_{1}^{n}, \cdots, F_{p n}^{n}
$$

a collection of pairwise disjoint cubes so that

if and only if

$$
F_{i}^{n} \subset \operatorname{Int} F_{j}^{n-1}
$$

$$
K_{i}^{n} \subset \operatorname{Int} K_{j}^{n-1} \text {. }
$$

Without loss we may require that $\delta\left(F_{i}^{n}\right)<1 / n$. 
The following lemma is stated without proof.

Lemma. If $Q_{1}, \cdots, Q_{n}$ are disjoint polyhedral 2-spheres in $S^{3}$, no one interior to any other, and if $Q_{0}$ is a large polyhedral cube containing $Q_{1}, \cdots, Q_{n}$ in its interior, the closed domain bounded by $Q_{0}, Q_{1}, \cdots, Q_{n}$ is tame. Further, any two domains so formed in this way are homeomorphic.

4. Let $P^{0}$ be a large cube in $S^{3}$ containing $\widetilde{P}_{1}, \cdots, \widetilde{P}_{p_{1}}$ in its interior. Then $K^{0}=f\left(P^{0}\right)$ is a 2 -sphere in $M$ containing $\tilde{K}_{1}^{\prime}, \cdots, \tilde{K}_{p_{1}}^{\prime}$ in its interior. Let $M_{0}$ be the region in $M$ exterior to $K^{0}$. Then $\bar{M}_{0}$ is homeomorphic to $S^{3} \backslash \operatorname{Int} P^{0}$ under $f^{-1}$. Hence there is a homeomorphism $h_{0}$ from $\bar{M}_{0}$ to $S^{3} \backslash$ Int $F_{0}$. Let $M_{1}=$ region in $M$ bounded by $K^{0} \cup \cup_{1}^{p_{1}} \tilde{K}_{i}^{\prime}$. Then, by the lemma, $\bar{M}_{1}$ is homeomorphic to the region in $S^{3}$ bounded by $F^{0} \cup \cup_{1}^{p_{1}} F_{i}^{\prime}$. Let $h_{1}$ be a homeomorphic extension of $h_{0}$ from $\bar{M}_{0}$ to $\bar{M}_{0} \cup \bar{M}_{1}$. The next step is similar, except that $M_{2}$ is a union of a finite number of regions bounded by the sets

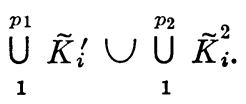

However, these regions are in 1-1 correspondence with the number of regions bounded by

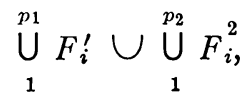

hence, by the lemma, the extension of $h_{1}$ from $\bar{M}_{0} \cup \bar{M}_{1}$ to $\bar{M}_{0} \cup \bar{M}_{1}$ $\cup \bar{M}_{2}$ can be carried out.

Continuing, a sequence of homeomorphisms $h_{0}, h_{1}, h_{2}, \ldots$ is defined so that each is an extension of the preceding and

$$
h(x)=h_{n}(x)
$$

maps $M \backslash \bar{Y}$ homeomorphically onto the complement of a Cantor set $X$ in $S^{3}$.

Since nested sequences of connected sets in $M \backslash \bar{Y}$ correspond to nested sequences of connected sets in $S^{3} \backslash X$, it is easy to see that $h$ and $h^{-1}$ are both uniformly continuous, hence the extension $\tilde{h}$ of $h$ carries $M$ homeomorphically onto $S^{3}$.

\section{REFERENCES}

1. R. H. Bing, $A$ decomposition of $E^{3}$ into points and tame arcs such that the decomposition space is topologically different from $E^{3}$, Ann. of Math. vol. 65, no. 3 (1957) pp. 484-500.

2. R. H. Bing, Approximating surfaces with polyhedral ones. Ann. of Math. vol. 65, no. 3 (1957) pp. 456-483.

3. R. L. Moore, Concerning upper semi-continuous collections of continua. Trans. Amer. Math. Soc., vol. 27 (1925) pp. 416-428.

The University of Tennessee and

The Institute for Advanced Study 